\title{
Regional Nerve Block Decreases the Incidence of Postoperative Delirium in Elderly Hip Fracture
}

\author{
Eic Ju Lim ${ }^{1}$, Won Uk Koh $\left.{ }^{2}{ }^{(}\right)$, Hyungtae Kim ${ }^{2}{ }^{(0)}$, Ha-Jung Kim ${ }^{2}$, Hyun-Chul Shon ${ }^{1}$ and Ji Wan Kim ${ }^{3, *}$ \\ 1 Department of Orthopedic Surgery, Chungbuk National University Hospital, Chungbuk National University \\ College of Medicine, Cheongju 28644, Korea; limeicju@gmail.com (E.J.L.); \\ hyunchuls@chungbuk.ac.kr (H.-C.S.) \\ 2 Department of Anesthesiology and Pain Medicine, Asan Medical Center, University of Ulsan College of \\ Medicine, Seoul 05505, Korea; koh9726@naver.com (W.U.K.); ingwei2475@gmail.com (H.K.); \\ Alexakim06@gmail.com (H.-J.K.) \\ 3 Department of Orthopedic Surgery, Asan Medical Center, University of Ulsan College of Medicine, \\ Seoul 05505, Korea \\ * Correspondence: bakpaker@hanmail.net; Tel.: +82-2-3010-3530
}

Citation: Lim, E.J.; Koh, W.U.; Kim, H.; Kim, H.-J.; Shon, H.-C.; Kim, J.W. Regional Nerve Block Decreases the Incidence of Postoperative Delirium in Elderly Hip Fracture. J. Clin. Med. 2021, 10, 3586. https://doi.org/ $10.3390 / \mathrm{jcm} 10163586$

Academic Editor: Gianluca Testa

Received: 21 July 2021

Accepted: 12 August 2021

Published: 15 August 2021

Publisher's Note: MDPI stays neutral with regard to jurisdictional claims in published maps and institutional affiliations.

Copyright: (C) 2021 by the authors. Licensee MDPI, Basel, Switzerland. This article is an open access article distributed under the terms and conditions of the Creative Commons Attribution (CC BY) license (https:// creativecommons.org/licenses/by/ $4.0 /)$.

\begin{abstract}
Postoperative delirium is common in elderly patients with hip fracture. Pain is a major risk factor for delirium, and regional nerve blocks (RNBs) effectively control pain in hip fractures. This study aimed to evaluate the effect of RNB on delirium after hip surgery in elderly patients. This retrospective comparative study was performed in a single institution, and the data were collected from medical records between March 2018 and April 2021. Patients aged $\geq 60$ years who underwent proximal femoral fracture surgery were included, while those with previous psychiatric illness and cognitive impairment were excluded. Two hundred and fifty-two patients were enrolled and divided into an RNB or a control group according to RNB use. Delirium was assessed as the primary outcome and postoperative pain score, pain medication consumption, and rehabilitation assessment as the secondary outcomes. Between the RNB $(n=129)$ and control groups $(n=123)$, there was no significant difference in the baseline characteristics. The overall incidence of delirium was $21 \%$; the rate was lower in the RNB group than in the control group (15 vs. $27 \%$, respectively, $p=0.027$ ). The average pain score at $6 \mathrm{~h}$ postoperatively was lower in the RNB group than in the control group $(2.8 \pm 1.5$ vs. $3.3 \pm 1.6$, respectively, $p=0.030)$. There was no significant difference in the pain score at 12,24 , and $48 \mathrm{~h}$ postoperatively, amount of opioids consumed for 2 postoperative days, and time from injury to wheelchair ambulation. We recommend RNB as a standard procedure for elderly patients with hip fracture due to lower delirium incidence and more effective analgesia in the early postoperative period.
\end{abstract}

Keywords: hip fracture; nerve block; delirium; pain; opioids

\section{Introduction}

Osteoporotic hip fracture is a major health problem because it is associated with high mortality, morbidity, and costs [1]. Although there are downward trends of mortality related to hip fracture, greater efforts are needed to achieve better outcomes [2]. The health status and health-related quality of life of elderly patients are seriously affected by the presence of hip fracture, and most patients cannot return to their performance status before injury [3].

Postoperative delirium is one of the most common complications in elderly patients with hip fracture and could result in cognitive impairment, short-term functional impairment, and increased mortality [4]. The known predisposing factors for delirium include advanced age, hip fracture surgery (in comparison to elective hip surgery), preoperative psychiatric illness, and preoperative cognitive impairment [5]. In addition, pain is a major risk factor for delirium; however, most elderly patients with hip fracture have a limited 
use of systemic opioid analgesics owing to side effects and their vulnerability in the drug metabolism process [6,7].

For this reason, comprehensive pain protocols have been suggested for elderly patients with hip fracture; these include evidence-based block use, timely repeated pain assessment, and multidisciplinary orthogeriatric care [8]. Regional nerve blocks (RNBs) have been proven to be effective in controlling pain in hip fractures, along with the advantages of few systemic effects [9]. Considering that pain control is essential for the reduction in delirium, we hypothesized that RNB use in hip fracture surgery would decrease postoperative pain and the incidence of delirium. This study aimed to evaluate the incidence of delirium according to RNB use in patients with hip fracture.

\section{Materials and Methods}

\subsection{Study Population}

This retrospective comparative study was performed in a single institution and approved by our institutional review board. The data were collected from medical records between March 2018 and April 2021. The inclusion criteria were as follows: (1) age of $\geq 60$ years and (2) surgical treatment of proximal femoral fracture, which was defined as a femoral neck fracture, an intertrochanteric femoral fracture (AO/OTA 31) [10], and a subtrochanteric fracture (fracture extending $5 \mathrm{~cm}$ below the lower border of the lesser trochanter) [11]. Meanwhile, the exclusion criteria were as follows: (1) previous psychiatric illness, (2) previous cognitive impairment, (3) pathologic fracture, (4) prophylactic fixation, (5) revision of total hip replacement, (6) delayed surgery with neglected fracture, and (7) incomplete clinical data. Initially, 307 patients were included; ultimately, 252 patients were enrolled in this study. The patients who received general or spinal anesthesia followed by RNB were grouped into the RNB group. The patients who received only general or spinal anesthesia were grouped into the control group (Figure 1).

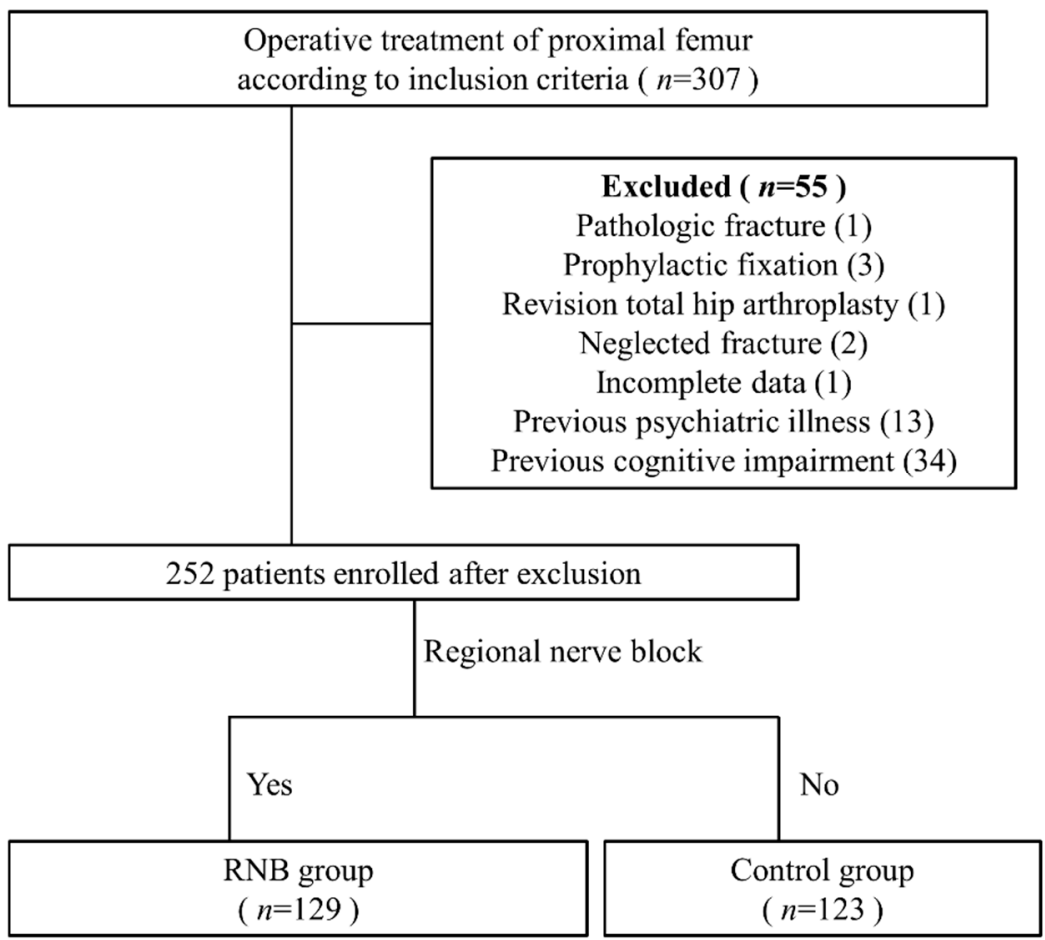

Figure 1. Flow diagram of patient enrollment and grouping.

\subsection{Procedures}

General or spinal anesthesia was performed according to the patients' overall health status, and RNB use was left to the discretion of the anesthesiologist. Under ultrasound guidance, a single shot of RNB, including fascia iliaca compartment block (FICB) [12], 
lumbar plexus block (LPB) [13], and pericapsular nerve group (PENG) block [14], was applied (Figure 2). For ultrasound-guided RNBs, a transportable ultrasound with $60 \mathrm{~mm}$ convex 2-5 MHz transducer for LPB and $25 \mathrm{~mm}$ linear 18-4 MHz transducer (Sonimage HS1TM, Konica Minolta Inc., Wayne, NJ, USA), and 21-gauge echoplex needle (Vygon, Ecouen, France) were used. After determining the insertion site, real-time ultrasoundguided perineural injection was conducted with the patient in supine or lateral position. Then, $30 \mathrm{~mL}$ of $0.3 \%$ ropivacaine was administered for LPB and FICB. Additionally, $20 \mathrm{~mL}$ of $0.3 \%$ ropivacaine was administered for PENG block.

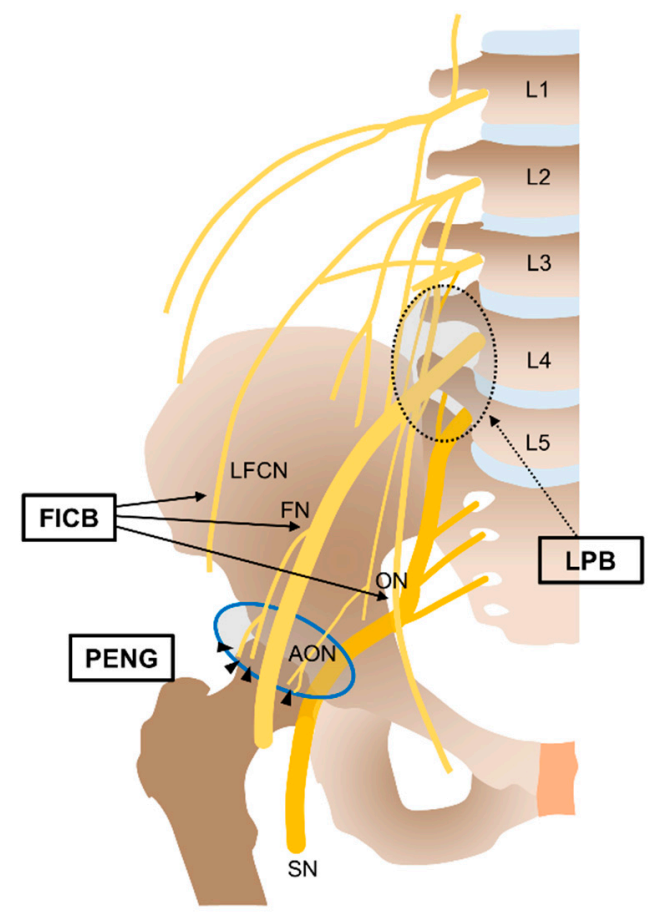

Figure 2. Illustration of the target nerves according to the type of regional nerve block. The fascia iliaca compartment block (FICB) targets the lateral femoral cutaneous (LFCN), femoral (FN), and obturator nerves (ON). The lumbar plexus block (LPB) targets the lumbar plexus (FN, ON, and LFCN) (dotted circle). The pericapsular nerve group (PENG) block mainly targets the articular branches (black arrowhead) of the FN and accessory obturator nerve (AON) (blue solid circle). SN: Sciatic nerve.

The patients with femoral neck fracture underwent bipolar hemiarthroplasty or internal fixation with multiple screws, while those with intertrochanteric and subtrochanteric fractures underwent intramedullary nailing. After surgery, intravenous patient-controlled analgesia (PCA) was applied in all patients. On the day of surgery, intravenous acetaminophen $1 \mathrm{~g}$ once a day was administered unless the patient had a contraindication. If the weight of the patient was less than $50 \mathrm{~kg}$, the dose of acetaminophen was controlled at $15 \mathrm{mg} / \mathrm{kg}$. The day after surgery, oral pain medications included a tramadol $37.5 \mathrm{mg}$ /acetaminophen $325 \mathrm{mg}$ tablet given twice daily and tapentadol $100 \mathrm{mg}$ given twice daily. Rescue analgesia during postoperative period was intravenous tramadol $50 \mathrm{mg}$ or hydromorphone $0.5 \mathrm{or} 1.0 \mathrm{mg}$. Periarticular injection was not performed. We encouraged wheelchair ambulation as soon as possible from the day after surgery, and tolerable weight-bearing ambulation started on 2 days postoperatively.

\subsection{Data Collection}

Demographic data were collected from the patients' medical records, including age, sex, body mass index (BMI), age-adjusted Charlson comorbidity index (ACCI), Koval score before injury, injury mechanism, anesthesia method, fracture type, time from injury to 
surgery, time from admission to surgery, and type of surgery. The Charlson comorbidity index (CCI) was calculated by adding the coefficient assigned to comorbidities when injured. The ACCI was calculated by adding 1 point for each decade after the age of 40 years to the CCI value [15]. Falls from heights of $1 \mathrm{~m}$ or less were defined as "low-energy mechanism of injury" [16].

Delirium was recorded and defined using the confusion assessment method on any postoperative day or night of their hospital stay following surgery as the primary outcome [17]. Postoperative pain score, pain medication consumption, and rehabilitation assessment were evaluated as the secondary outcomes. The pain scores at $6,12,24$, and $48 \mathrm{~h}$ postoperatively were assessed using the visual analog scale pain score. The consumption of pain medication for 2 postoperative days was examined. The amount of analgesics was calculated into milligrams of oral morphine according to the equianalgesic table [18-20]. A rehabilitation assessment was performed on the basis of the time (days) from surgery to wheelchair ambulation. All these parameters (pain score, incidence of delirium, pain medication consumption, and rehabilitation assessment) were included in the standardized protocol of our hospital.

The primary and secondary outcomes were compared between the RNB and control groups. A subgroup analysis for incidence of postoperative delirium between general and spinal anesthesia was performed. A subgroup analysis between FICB, LPB, and PENG block was performed to evaluate the differences between the blocks.

A multivariable analysis was performed to assess which variables were associated with incidence of delirium and clinically significant parameters were included in the model such as age, gender, CCI, RNB, and anesthesia type [21-23]. Nerve block-related complications, such as falls within $48 \mathrm{~h}$ after surgery and nerve injury, were assessed from the medical records. Postoperative medical complications, including deep vein thrombosis, pulmonary embolism, pneumonia, angina, myocardial infarction, and urinary tract infection, were evaluated.

\subsection{Statistical Analysis}

Categorical variables (e.g., sex, injury mechanism, method of anesthesia, fracture type, type of surgery, and incidence of delirium) were analyzed using the chi-square or Fisher's exact test. Continuous variables (e.g., age, BMI, ACCI, Koval score, time from injury to surgery, pain score, amount of analgesics consumed, and time to wheelchair ambulation) were analyzed using an independent t-test or the Mann-Whitney test. The Shapiro-Wilk test was used to check if the data distribution was normal. The Kruskal-Wallis test was used when compare more than two continuous variables. A logistic regression analysis was conducted for multivariable analysis. All continuous data are described as means and standard deviations. Statistical significance was accepted for $p$-values of $<0.05$ using SPSS version 23.0 (IBM Corp., Armonk, NY, USA).

\section{Results}

The RNB group consisted of 129 patients, while the control group consisted of 123 patients. The baseline characteristics of the two study groups are presented in Table 1. There was no significant difference found in the average age, BMI, ACCI, Koval score before injury, injury mechanism, method of anesthesia, fracture type, time from injury to surgery, time from admission to surgery, and type of surgery. The methods of RNB are summarized in Table 2. 
Table 1. Baseline characteristics of the RNB and control groups.

\begin{tabular}{|c|c|c|c|}
\hline & $\begin{array}{l}\text { RNB Group } \\
\quad(n=129)\end{array}$ & $\begin{array}{l}\text { Control Group } \\
\quad(n=123)\end{array}$ & $p$-Value \\
\hline Age (years) & $78.1 \pm 8.3$ & $77.6 \pm 8.8$ & 0.646 \\
\hline \multicolumn{4}{|l|}{ Sex } \\
\hline Male & $32(25 \%)$ & $40(32 \%)$ & 0.175 \\
\hline Female & $97(75 \%)$ & $83(68 \%)$ & \\
\hline BMI & $22.4 \pm 3.7$ & $22.7 \pm 3.8$ & 0.560 \\
\hline $\mathrm{ACCI}$ & $6.3 \pm 2.0$ & $5.9 \pm 1.8$ & 0.108 \\
\hline Koval score before injury * & $1.9 \pm 1.5$ & $2.1 \pm 1.7$ & 0.238 \\
\hline \multicolumn{4}{|l|}{ Injury mechanism } \\
\hline Low energy & $125(97 \%)$ & $117(95 \%)$ & 0.532 \\
\hline High energy & $4(3 \%)$ & $6(5 \%)$ & \\
\hline \multicolumn{4}{|l|}{ Anesthesia method } \\
\hline General & $60(46 \%)$ & $58(47 \%)$ & 1.000 \\
\hline Spinal & $69(54 \%)$ & $65(53 \%)$ & \\
\hline \multicolumn{4}{|l|}{ Fracture type } \\
\hline Femoral neck fracture & $53(41 \%)$ & $58(47 \%)$ & 0.611 \\
\hline Pertrochanteric fracture & $63(49 \%)$ & $53(43 \%)$ & \\
\hline Subtrochanteric fracture & $13(10 \%)$ & $12(10 \%)$ & \\
\hline Time from injury to surgery (hour) $^{\dagger}$ & $89.7 \pm 99.4$ & $111.0 \pm 164.1$ & 0.197 \\
\hline Time from admission to surgery (hour) & $59.4 \pm 68.7$ & $62.6 \pm 96.7$ & 0.757 \\
\hline \multicolumn{4}{|l|}{ Type of surgery } \\
\hline Osteosynthesis & $88(68 \%)$ & $76(62 \%)$ & 0.285 \\
\hline Arthroplasty & $41(32 \%)$ & $47(38 \%)$ & \\
\hline
\end{tabular}

RNB, regional nerve block; BMI, body mass index; ACCI, age-adjusted Charlson comorbidity index; * calculated from 249 patients who had clinical records on the preoperative Koval score; ${ }^{\dagger}$ calculated from 246 patients who had clinical records on the time from injury to surgery.

Table 2. Type of RNB for hip fracture.

\begin{tabular}{cl}
\hline Type of RNB & \\
\hline Fascia iliaca compartment block & $78(60 \%)$ \\
Lumbar plexus block & $29(23 \%)$ \\
Pericapsular nerve group block & $22(17 \%)$ \\
\hline
\end{tabular}

RNB, regional nerve block.

The overall incidence of delirium was $21 \%$; the rate in the RNB group was lower than that in the control group ( 15 vs. $27 \%, p=0.027$ ). There were no nerve block-related complications, including falls within $48 \mathrm{~h}$ after surgery and nerve injury.

The average pain score at $6 \mathrm{~h}$ postoperatively in the RNB group was lower than that in the control group $(2.8 \pm 1.5$ vs. $3.3 \pm 1.6$, respectively, $p=0.030)$ (Table 3$)$. There was no significant difference in the pain score at 12 (RNB group: $2.8 \pm 1.6$ vs. control group: $2.7 \pm 1.5, p=0.432$ ), 24 (RNB group: $2.5 \pm 1.3$ vs. control group: $2.4 \pm 1.4, p=0.154$ ), and 48 (RNB group: $2.2 \pm 1.1$ vs. control group: $2.0 \pm 1.1, p=0.083$ ) hours postoperatively. There was also no significant difference in the amount of opioids consumed for 2 postoperative days (oral morphine; RNB group: $36.2 \pm 25.4 \mathrm{mg}$ vs. control group: $33.5 \pm 28.0 \mathrm{mg}$, $p=0.322$ ) and time from injury to wheelchair ambulation (RNB group: $1.8 \pm 2.4$ days vs. control group: $1.7 \pm 1.1$ days, $p=0.407$ ).

In the subgroup analysis between general and spinal anesthesia, there was no significant difference in the incidence of postoperative delirium (Table 4). In the subgroup analysis between FICB, LPB, and PENG block, there was no significant difference in the incidence of postoperative delirium, pain score, and amount of opioids consumed, but time to wheelchair ambulation presented a significant difference for PENG block compared with FICB and LPB (Table 5). 
Table 3. Comparison of the postoperative pain score, incidence of delirium, amount of opioids consumed, and time to wheelchair ambulation.

\begin{tabular}{cccc}
\hline & $\begin{array}{c}\text { RNB Group } \\
(\boldsymbol{n}=\mathbf{1 2 9})\end{array}$ & $\begin{array}{c}\text { Control Group } \\
(\boldsymbol{n}=\mathbf{1 2 3})\end{array}$ & $p$-Value \\
\hline Incidence of postoperative delirium & $20(15 \%)$ & $33(27 \%)$ & 0.027 \\
Postoperative pain score & & & \\
6 h postoperatively & $2.8 \pm 1.5$ & $3.3 \pm 1.6$ & 0.030 \\
12 h postoperatively & $2.8 \pm 1.6$ & $2.7 \pm 1.5$ & 0.432 \\
24 h postoperatively & $2.5 \pm 1.3$ & $2.4 \pm 1.4$ & 0.154 \\
48 h postoperatively & $2.2 \pm 1.1$ & $2.0 \pm 1.1$ & 0.083 \\
Amount of opioids consumed (mg) ${ }^{*}$ & $36.2 \pm 25.4$ & $33.5 \pm 28.0$ & 0.322 \\
Time to wheelchair ambulation (days) $^{+}$ & $1.8 \pm 2.4$ & $1.7 \pm 1.1$ & 0.407 \\
\hline
\end{tabular}

$\mathrm{RNB}$, regional nerve block; ${ }^{*}$ expressed as milligrams of oral morphine by equianalgesic conversion; ${ }^{\dagger}$ calculated from 247 patients who had clinical records on the time to wheelchair ambulation.

Table 4. Subgroup analysis for the incidence of delirium between general and spinal anesthesia.

\begin{tabular}{cccc}
\hline $\begin{array}{c}\text { Incidence of } \\
\text { Postoperative } \\
\text { Delirium }\end{array}$ & General Anesthesia & Spinal Anesthesia & $\boldsymbol{p}$-Value \\
\hline RNB group & $9 / 60(15.0 \%)$ & $11 / 69(15.9 \%)$ & 0.883 \\
Control group & $15 / 58(25.9 \%)$ & $18 / 65(27.7 \%)$ & 0.819 \\
Total & $24 / 118(20.3 \%)$ & $29 / 134(21.6 \%)$ & 0.800 \\
\hline
\end{tabular}

RNB, regional nerve block.

Table 5. Subgroup analysis for the postoperative pain score, incidence of delirium, amount of opioids consumed, and time to wheelchair ambulation between FICB, LPB and PENG block.

\begin{tabular}{ccccc}
\hline & $\begin{array}{c}\text { FICB } \\
(\boldsymbol{n}=\mathbf{7 8})\end{array}$ & $\begin{array}{c}\text { LPB } \\
(\boldsymbol{n}=\mathbf{2 9 )}\end{array}$ & $\begin{array}{c}\text { PENG Block } \\
(\boldsymbol{n}=\mathbf{2 2})\end{array}$ & $p$-Value \\
\hline Incidence of postoperative delirium & $13(17 \%)$ & $5(17 \%)$ & $2(9.1 \%)$ & 0.786 \\
Postoperative pain score & & & & \\
6 h postoperatively & $3.0 \pm 1.5$ & $2.4 \pm 1.6$ & $2.9 \pm 1.5$ & 0.215 \\
12 h postoperatively & $2.7 \pm 1.6$ & $2.7 \pm 1.7$ & $3.3 \pm 1.4$ & 0.264 \\
24 h postoperatively & $2.5 \pm 1.3$ & $2.4 \pm 1.4$ & $3.0 \pm 1.0$ & 0.161 \\
48 h postoperatively & $2.1 \pm 1.1$ & $2.3 \pm 1.4$ & $2.6 \pm 0.9$ & 0.118 \\
Amount of opioids consumed (mg) ${ }^{*}$ & $35.7 \pm 24.4$ & $33.6 \pm 29.2$ & $41.6 \pm 24.9$ & 0.372 \\
Time to wheelchair ambulation (days) $^{\dagger}$ & $1.7 \pm 1.2$ & $2.5 \pm 4.5$ & $1.0 \pm 0.2$ & 0.020 \\
\hline
\end{tabular}

FICB, fascia iliaca compartment block; LPB, lumbar plexus block; PENG block, pericapsular nerve group block; ${ }^{*}$ expressed as milligrams of oral morphine by equianalgesic conversion; ${ }^{\dagger}$ calculated from 106 patients who had clinical records on the time to wheelchair ambulation.

In the multivariable analysis, age and RNB were significantly associated with the incidence of postoperative delirium (Table 6). There was also no significant difference in the postoperative medical complications between them (Table 7).

Table 6. Bivariate and multivariable logistic regression analysis for the risk factors of postoperative delirium.

\begin{tabular}{ccccc}
\hline & \multicolumn{3}{c}{ Incidence of Postoperative Delirium } \\
\cline { 2 - 5 } & \multicolumn{2}{c}{ Bivariate Analysis } & \multicolumn{2}{c}{ Multivariable Logistic Regression * } \\
\hline Odds Ratio (95\% CI) & $p$-Value & Odds Ratio (95\% CI) & $p$-Value \\
Age & $1.060(1.020-1.101)$ & 0.003 & $1.062(1.022-1.104)$ & 0.002 \\
Female sex & $0.906(0.467-1.757)$ & 0.769 & \\
CCI & $1.091(0.934-1.275)$ & 0.270 & & 0.022 \\
RNB & $0.500(0.269-0.932)$ & 0.029 & $0.476(0.252-0.898)$ & \\
Spinal anesthesia & $1.082(0.589-1.978)$ & 0.800 & & \\
\hline
\end{tabular}

* The results of multivariable logistic regression analysis were presented only for the variables which were remained in the final model. 
Table 7. Postoperative complications.

\begin{tabular}{cccc}
\hline & $\begin{array}{c}\text { RNB Group } \\
(\boldsymbol{n}=\mathbf{1 2 9})\end{array}$ & $\begin{array}{c}\text { Control Group } \\
(\boldsymbol{n}=\mathbf{1 2 3})\end{array}$ & $p$-Value \\
\hline Deep vein thrombosis & $1(1 \%)$ & $1(1 \%)$ & $>0.999$ \\
Pulmonary embolism & $1(1 \%)$ & $1(1 \%)$ & $>0.999$ \\
Pneumonia & $5(4 \%)$ & $4(3 \%)$ & $>0.999$ \\
Angina or myocardial infarction & $1(1 \%)$ & $3(2 \%)$ & 0.360 \\
Urinary tract infection & $4(3 \%)$ & $3(2 \%)$ & $>0.999$ \\
\hline
\end{tabular}

RNB, regional nerve block.

\section{Discussion}

\subsection{Incidence of Delirium}

In this study, RNB reduced the incidence of delirium. It is widely accepted that pain reduction is important for the prevention of delirium [6], and we hypothesized that postoperative pain control could prevent delirium after hip fracture surgery. Our study results proved the effect of RNB on the incidence of postoperative delirium after hip fracture surgery. Herein, we excluded patients with a high risk of developing delirium, including those with psychiatric illness and cognitive impairment, which was based on a study by Mouzopoulos et al. [22]. They classified patients into intermediate or high risk for postoperative delirium, and FICB did not affect the incidence of delirium in patients with high risk after hip fracture surgery. In contrast, a significant reduction in the incidence of delirium (FICB group vs. placebo group: 2.4 vs. $16.9 \%$ ) was observed among patients with an intermediate in their study.

The results of RNB and delirium in patients with hip fracture are inconsistent. RNB was reported to be associated with less postoperative analgesia, a lower incidence of delirium, and shorter inpatient stay $[24,25]$. In contrast, Guay et al. presented that there were no differences in the incidence of acute confusional state in their Cochrane review based on seven trials with 676 participants [26]. However, the study has a limitation of heterogeneity and lack of risk stratification. Unneby et al. reported that femoral nerve block (FNB) did not reduce the incidence of postoperative delirium in patients with hip fracture [27]. They focused on patients with dementia, and a large proportion developed delirium (FNB group vs. placebo group: 50/52 vs. 55/57) regardless of FNB use. This suggests that patients with major risk factors, such as cognitive and psychiatric disorders, are highly prone to developing delirium regardless of pain control. In the present study, we could prove the effect of RNB on the incidence of delirium by excluding high risk patients with the inclusion of a relatively large number of patients as a single study. Additionally, we performed a multivariable analysis with other known risk factors of delirium [21-23]. Age and RNB were presented to be associated with postoperative delirium, which means an appropriate risk stratification of present study. Therefore, we believe the RNB helps to prevent postoperative delirium after hip fracture surgery except in high risk elderly patients.

\subsection{Effect on Pain Intensity and Opioid Consumption}

Opioids are useful in reducing pain after surgery but have limitations regarding side effects and drug poisoning [28]. Elderly patients are known to be vulnerable to the side effects of opioids, with reductions in renal and hepatic blood flow [29]; in our study, one of our purposes was the reduction in opioid consumption with the effect of nerve block. Thompson et al. reported that preoperative fascia iliaca block significantly decreased postoperative opioid consumption [30]. They reduced the amount of tramadol by $43 \%$ and morphine by $98 \%$ with fascia iliaca block. However, contrary to our expectations, there was no significant difference in the amount of opioids consumed despite early pain reduction in our study. The first possible reason is that diverse types of opioids were prescribed with a retrospective feature. Although we calculated the equianalgesic dose of each opioid into milligrams of oral morphine [18-20], the results could be influenced by the diversity of the opioid types prescribed. The second possible reason is that all patients received 
intravenous PCA because it is a customary procedure desired by patients. In addition to additional opioids injected by nurses, rescue opioids were also administered through PCA, which were not included in the calculation of the quantity of opioids consumed. These reasons may have influenced the outcome for the quantity of opioids.

\subsection{Functional Recovery}

Based on the results reported by Marino et al., who demonstrated that continuous lumbar plexus block provided pain reduction during physical therapy [31], we expected that functional recovery could be encouraged with nerve block. However, there was no significant difference observed in the time to wheelchair ambulation between the two groups, which can be explained by some reasons. A consistent rehabilitation protocol was applied to the patients in both groups. In addition, Kim et al. demonstrated that the postoperative ambulatory capacity after hip fracture surgery is decided not by only a single factor but by multiple factors, including age, sex, preoperative ambulatory capacity, and combined medical diseases [32]. Since the postoperative ambulatory capacity is significantly associated with preoperative factors, it is possible that the reduction in pain itself could not affect the short-term functional recovery.

\subsection{Subgroup Analysis According to Type of RNB and Anesthesia Method}

In clinical practice, we usually perform LPB after induction of general anesthesia because it should be conducted in the lateral decubitus position. In contrast, FICB is usually performed before induction of spinal anesthesia because it could be conducted in the supine position, which could reduce pain for positioning of spinal anesthesia. Since LPB and FICB could block both anterior innervations of the hip joint and some surgical incision site, similar pain reduction and delirium prevention could be expected in both blocks (Figure 2). In our study, the subgroup analysis between RNBs did not demonstrate significant differences in the postoperative pain score and amount of opioids consumed, but time to wheelchair ambulation of PENG block presented significant differences compared to that of FICB and LPB. The possible reason for these results was that PENG block was introduced as a potential motor sparing analgesic block [14,33], which could encourage patients to ambulate early. However, investigation for PENG block is very limited, and validation to propose motor sparing and analgesic benefit is needed [33]. Additionally, with a relatively small number of patients included in each RNBs in the present study, detailed analysis according to block type requires careful interpretation.

There could be concerns regarding anesthesia method. Previous studies showed controversial results. Choi et al. reported general anesthesia was an independent predictor of immediate delirium [23]. In contrast, Patel et al. concluded that there was no evidence to suggest that anesthesia type influence postoperative delirium in their systematic review [34]. In the present study, there were no significant differences in the incidence of delirium between general and spinal anesthesia in subgroup analysis, and multivariate analysis showed no effect on postoperative delirium according to anesthesia method.

\subsection{Procedure-Related Complications}

Falls and procedure-related nerve injuries are important complications of RNB in patients with hip fracture [35,36]; in our study, no complication was observed. While wheelchair ambulation was encouraged from the day after surgery, the protocol of our institution was to involve at least three individuals (nurse, caregiver, and paramedics) in the transfer of a patient to a wheelchair. At 2 days postoperatively, the patients were permitted to bear weight as tolerable. Considering that the duration of a single shot of bupivacaine $0.5 \%(20 \mathrm{~mL})$ is 22 (range, 15-32) hours [37], it is unlikely that a fall would occur during ambulation owing to RNB. Further, all RNBs were performed by experienced anesthesiologists under ultrasound guidance. We believe that our rehabilitation protocol and technique for RNB could ensure the safeness of RNB in patients with hip fracture. 


\subsection{Study Limitations}

There were some limitations in this study. First, our study had a selection bias in relation to the retrospectively evaluated characteristics. RNB use was decided by the anesthesiologists, and there was no clear criterion. Future prospective studies with randomization are needed. Second, the types of nerve block used varied. FICB, LSPB, and PENG block were used herein, which might have confounded the results. Third, the severity of delirium was not considered. Some patients only demonstrated temporary inattention; however, other patients demonstrated irritability that needed restraint. We classified these demonstrations as delirium. Subgroup analyses according to the severity of delirium will be helpful in the evaluation of the effects of RNB. Despite these limitations, our study provided evidence that RNB can reduce the incidence of delirium, and we believe that its use can help improve the prognosis of elderly patients with hip fracture.

\section{Conclusions}

RNB reduced the occurrence of delirium in elderly patients with hip fracture and relieved acute pain after surgery without complications. Therefore, we recommend RNB as a standard procedure for elderly patients with hip fracture due to lower delirium incidence and more effective analgesia in the early postoperative period.

Author Contributions: Writing—original draft, formal analysis, visualization, E.J.L.; Investigation and resources, data curation, W.U.K.; Investigation and resources, H.K.; Validation, project administration, H.J.K.; Methodology, supervision, H.-C.S.; Conceptualization, investigation and resources, writing-review and editing, J.W.K. All authors have read and agreed to the published version of the manuscript.

Funding: This research was supported by the Technology Innovation Program (20000397, Development of Two Track Customized 3D Printing Implant Manufacturing and Commercializing Techniques for Complex Bone Fractures) funded by the Ministry of Trade, Industry and Energy (MOTIE, Korea).

Institutional Review Board Statement: The study was conducted according to the guidelines of the Declaration of Helsinki and approved by the Institutional Review Board of ASAN MEDICAL CENTER (protocol no. 2019-0267; December, 2019).

Informed Consent Statement: Patient consent was waived owing to the retrospective nature of this study.

Data Availability Statement: The data presented in this study are available on request from the corresponding author. The data are not publicly available due to conditions of the ethics committee of our university.

Conflicts of Interest: The authors declare no conflict of interest.

\section{References}

1. Braithwaite, R.S.; Col, N.F.; Wong, J.B. Estimating hip fracture morbidity, mortality and costs. J. Am. Geriatr. Soc. 2003, 51, 364-370. [CrossRef]

2. Orces, C.H. Hip Fracture-Related Mortality among Older Adults in the United States: Analysis of the CDC WONDER Multiple Cause of Death Data, 1999-2013. Epidemiol. Res. Int. 2016, 2016, 8970259. [CrossRef]

3. Alexiou, K.I.; Roushias, A.; Varitimidis, S.E.; Malizos, K.N. Quality of life and psychological consequences in elderly patients after a hip fracture: A review. Clin. Interv. Aging 2018, 13, 143-150. [CrossRef]

4. Lee, H.B.; Oldham, M.A.; Sieber, F.E.; Oh, E.S. Impact of Delirium After Hip Fracture Surgery on One-Year Mortality in Patients With or Without Dementia: A Case of Effect Modification. Am. J. Geriatr. Psychiatry 2017, 25, 308-315. [CrossRef]

5. Rizk, P.; Morris, W.; Oladeji, P.; Huo, M. Review of Postoperative Delirium in Geriatric Patients Undergoing Hip Surgery. Geriatr. Orthop. Surg. Rehabil. 2016, 7, 100-105. [CrossRef] [PubMed]

6. Morrison, R.S.; Magaziner, J.; Gilbert, M.; Koval, K.J; McLaughlin, M.A.; Orosz, G.; Strauss, E.; Siu, A.L. Relationship between pain and opioid analgesics on the development of delirium following hip fracture. J. Gerontol. Ser. A Biol. Sci. Med. Sci. 2003, 58, 76-81. [CrossRef]

7. Nie, H.; Zhao, B.; Zhang, Y.Q.; Jiang, Y.H.; Yang, Y.X. Pain and cognitive dysfunction are the risk factors of delirium in elderly hip fracture Chinese patients. Arch. Gerontol. Geriatr. 2012, 54, e172-e174. [CrossRef] [PubMed]

8. Scurrah, A.; Shiner, C.T.; Stevens, J.A.; Faux, S.G. Regional nerve blockade for early analgesic management of elderly patients with hip fracture-a narrative review. Anaesthesia 2018, 73, 769-783. [CrossRef] [PubMed]

9. Godoy Monzón, D.; Vazquez, J.; Jauregui, J.R.; Iserson, K.V. Pain treatment in post-traumatic hip fracture in the elderly: Regional block vs. systemic non-steroidal analgesics. Int. J. Emerg. Med. 2010, 3, 321-325. [CrossRef] 
10. Meinberg, E.G.; Agel, J.; Roberts, C.S.; Karam, M.D.; Kellam, J.F. Fracture and Dislocation Classification Compendium-2018. J. Orthop. Trauma 2018, 32 (Suppl. 1), S1-S170. [CrossRef] [PubMed]

11. Loizou, C.L.; McNamara, I.; Ahmed, K.; Pryor, G.A.; Parker, M.J. Classification of subtrochanteric femoral fractures. Injury 2010, 41, 739-745. [CrossRef]

12. Godoy Monzon, D.; Iserson, K.V.; Vazquez, J.A. Single fascia iliaca compartment block for post-hip fracture pain relief. J. Emerg. Med. 2007, 32, 257-262. [CrossRef]

13. Amiri, H.R.; Zamani, M.M.; Safari, S. Lumbar plexus block for management of hip surgeries. Anesth Pain Med. 2014,4 , e19407. [CrossRef]

14. Girón-Arango, L.; Peng, P.W.H.; Chin, K.J.; Brull, R.; Perlas, A. Pericapsular Nerve Group (PENG) Block for Hip Fracture. Reg. Anesth. Pain Med. 2018, 43, 859. [CrossRef] [PubMed]

15. Charlson, M.; Szatrowski, T.P.; Peterson, J.; Gold, J. Validation of a combined comorbidity index. J. Clin. Epidemiol. 1994, 47, 1245-1251. [CrossRef]

16. Bergstrom, U.; Bjornstig, U.; Stenlund, H.; Jonsson, H.; Svensson, O. Fracture mechanisms and fracture pattern in men and women aged 50 years and older: A study of a 12-year population-based injury register, Umea, Sweden. Osteoporos. Int. 2008, 19, 1267-1273. [CrossRef] [PubMed]

17. Inouye, S.K.; van Dyck, C.H.; Alessi, C.A.; Balkin, S.; Siegal, A.P.; Horwitz, R.I. Clarifying confusion: The confusion assessment method. A new method for detection of delirium. Ann. Intern. Med. 1990, 113, 941-948. [CrossRef]

18. Farrington, C.; Palliative Care Expert Group. Therapeutic Guidelines: Palliative Care, 3rd ed.; Therapeutic Guidelines Limited: Melbourne, Australia, 2010. [CrossRef]

19. Twycross, R.; Wilcock, A. PCF3: Palliative Care Formulary; Russell House Publishing: Nottingham, UK, 2007.

20. Gippsland Region Palliative Care Consortium. Opioid Conversion Guidelines; GRPCC: Melbourne, Australia, 2011.

21. Yang, Y.; Zhao, X.; Dong, T.; Yang, Z.; Zhang, Q.; Zhang, Y. Risk factors for postoperative delirium following hip fracture repair in elderly patients: A systematic review and meta-analysis. Aging Clin. Exp. Res. 2017, 29, 115-126. [CrossRef]

22. Mouzopoulos, G.; Vasiliadis, G.; Lasanianos, N.; Nikolaras, G.; Morakis, E.; Kaminaris, M. Fascia iliaca block prophylaxis for hip fracture patients at risk for delirium: A randomized placebo-controlled study. J. Orthop. Traumatol. 2009, 10, 127-133. [CrossRef] [PubMed]

23. Choi, Y.H.; Kim, D.H.; Kim, T.Y.; Lim, T.W.; Kim, S.W.; Yoo, J.H. Early postoperative delirium after hemiarthroplasty in elderly patients aged over 70 years with displaced femoral neck fracture. Clin. Interv. Aging 2017, 12, 1835-1842. [CrossRef]

24. Callear, J.; Shah, K. Analgesia in hip fractures. Do fascia-iliac blocks make any difference? BMJ Qual. Improv. Rep. 2016, 5, u210130.w214147. [CrossRef]

25. Del Rosario, E.; Esteve, N.; Sernandez, M.J.; Batet, C.; Aguilar, J.L. Does femoral nerve analgesia impact the development of postoperative delirium in the elderly? A retrospective investigation. Acute Pain 2008, 10, 59-64. [CrossRef]

26. Guay, J.; Parker, M.J.; Griffiths, R.; Kopp, S.L. Peripheral Nerve Blocks for Hip Fractures: A Cochrane Review. Anesth. Analg. 2018, 126, 1695-1704. [CrossRef]

27. Unneby, A.; Svensson, P.O.; Gustafson, P.Y.; Lindgren, A.P.B.; Bergström, U.; Olofsson, P.B. Complications with focus on delirium during hospital stay related to femoral nerve block compared to conventional pain management among patients with hip fracture-A randomised controlled trial. Injury 2020, 51, 1634-1641. [CrossRef] [PubMed]

28. Helmerhorst, G.T.; Teunis, T.; Janssen, S.J.; Ring, D. An epidemic of the use, misuse and overdose of opioids and deaths due to overdose, in the United States and Canada: Is Europe next? Bone Jt. J. 2017, 99-b, 856-864. [CrossRef]

29. Chau, D.L.; Walker, V.; Pai, L.; Cho, L.M. Opiates and elderly: Use and side effects. Clin. Interv. Aging 2008, 3, 273-278. [CrossRef] [PubMed]

30. Thompson, J.; Long, M.; Rogers, E.; Pesso, R.; Galos, D.; Dengenis, R.C.; Ruotolo, C. Fascia Iliaca Block Decreases Hip Fracture Postoperative Opioid Consumption: A Prospective Randomized Controlled Trial. J. Orthop. Trauma 2020, 34, 49-54. [CrossRef] [PubMed]

31. Marino, J.; Russo, J.; Kenny, M.; Herenstein, R.; Livote, E.; Chelly, J.E. Continuous lumbar plexus block for postoperative pain control after total hip arthroplasty. A randomized controlled trial. J. Bone Jt. Surg. Am. Vol. 2009, 91, 29-37. [CrossRef]

32. Kim, J.L.; Jung, J.S.; Kim, S.J. Prediction of Ambulatory Status After Hip Fracture Surgery in Patients Over 60 Years Old. Ann. Rehabil. Med. 2016, 40, 666-674. [CrossRef]

33. Kim, D.H.; Kim, S.J.; Liu, J.; Beathe, J.; Memtsoudis, S.G. Fascial plane blocks: A narrative review of the literature. Reg. Anesth Pain Med. 2021, 46, 600-617. [CrossRef]

34. Patel, V.; Champaneria, R.; Dretzke, J.; Yeung, J. Effect of regional versus general anaesthesia on postoperative delirium in elderly patients undergoing surgery for hip fracture: A systematic review. BMJ Open 2018, 8, e020757. [CrossRef] [PubMed]

35. Johnson, R.L.; Kopp, S.L.; Hebl, J.R.; Erwin, P.J.; Mantilla, C.B. Falls and major orthopaedic surgery with peripheral nerve blockade: A systematic review and meta-analysis. Br. J. Anaesth 2013, 110, 518-528. [CrossRef] [PubMed]

36. Borgeat, A. Neurologic deficit after peripheral nerve block: What to do? Minerva Anestesiol. 2005, 71, $353-355$.

37. Cuvillon, P.; Nouvellon, E.; Ripart, J.; Boyer, J.C.; Dehour, L.; Mahamat, A.; L’Hermite, J.; Boisson, C.; Vialles, N.; Lefrant, J.Y.; et al. A comparison of the pharmacodynamics and pharmacokinetics of bupivacaine, ropivacaine (with epinephrine) and their equal volume mixtures with lidocaine used for femoral and sciatic nerve blocks: A double-blind randomized study. Anesth. Analg. 2009, 108, 641-649. [CrossRef] [PubMed] 